\title{
“AQUÍ NO HAY QUIEN VIVA”. SOBRE LAS CASAS-POZO EN LA PREHISTORIA DE ANDALUCÍA DURANTE EL IV Y EL III MILENIOS AC
}

\author{
"IT'S IMPOSSIBLE TO LIVE HERE". ON THE PIT-HOUSES OF ANDALUSIAN \\ PREHISTORY DURING THE IV ${ }^{T H}$ AND $I I^{T H}$ MILLENNIA BC
}

\author{
VÍCTOR JIMÉNEZ JÁIMEZ* \\ JOSÉ ENRIQUE MÁRQUEZ ROMERO**
}

\begin{abstract}
Resumen: En este artículo se expresa una opinión crítica sobre la interpretación tradicional de los considerados poblados de fosos del Neolítico y Edad del Cobre del sur de la Península Ibérica. Especialmente, se discute la argumentación que identifica como fondos de cabaña muchas de las estructuras excavadas en el terreno. Se aborda la cuestión desde dos aspectos arqueológicos centrales: la formación de su relleno arqueológico y su naturaleza arquitectónica.

Palabras clave: Neolítico, Calcolítico, Pozos, Casas-pozo, Recintos de fosos.
\end{abstract}

\section{INTRODUCCIÓN. LA FORMACIÓN DEL REGISTRO ARQUEOLÓGICO EN LOS POBLADOS DE FOSOS EN EL SUR DE LA PENÍNSULA IBÉRICA}

El problema de los llamados yacimientos o poblados de fosos es, esencialmente, un problema sobre la formación del registro arqueológico (Marquez 2007: 27). Tal contingencia explica las dificultades encontradas a la hora de interpretar estos lugares y la discusión abierta sobre el particular durante décadas (ver Márquez, 2001; Márquez y Fernández 2002; Márquez 2006b; 2007).

\footnotetext{
* Área de Prehistoria. Universidad de Málaga: vjjaimez@uma.es.
}

** Área de Prehistoria. Universidad de Málaga: jemarquez@uma.es
Abstract: In this article we express a critical opinion about the interpretation of Southern Iberian Neolithic and Copper Age ditched enclosures as settlements composed by dwelling-pits, based on two aspects: their architectural properties and the formation processes of their archaeological fill.

Key words: Neolithic, Copper Age, Pits, Dwelling-pits, Ditched enclosures.

Sintetizando, podríamos concretar que la formación del registro arqueológico en estos yacimientos ha sido explicada conforme a tres hipotéticos procesos distintos. El primero considera el relleno de las estructuras en negativo como resultado de la lenta y progresiva acumulación de sedimentos en su interior -lo que Schiffer (1987) denominó desechos primarios ${ }^{1}-$, como consecuencia de la costumbre de utilizar los pozos como viviendas, es decir, de habitarlos físicamente como fondos de cabañas semisubterráneas y/o áreas productivas, de manera que los restos de actividades domésticas y productivas se habrían ido acumulando y con el tiempo configurarían niveles culturales (p.e. Cabrero 1987; Hornos et. al. 1987;

1. Según Schiffer (1987), los desechos primarios son aquellos descartados y depositados en su lugar de uso, mientras que los secundarios son desplazados de su lugar de uso para ser depositados en otro lugar. 
Rivero et. al. 1989; Cabrero 1990; Cruz-Auñón y Rivero 1990; Lizcano et. al. 1991-1992; Santana 1993; CruzAuñón et. al. 1995; Lizcano 1999; Ruiz Gil y Ruiz Mata 1999; Román y Conlin 2001; Ruiz Gil y López Amador 2001; Conlin y Gómez Saucedo 2003).

Una segunda interpretación consiste, por el contrario, en defender que las estructuras se colmataron de forma rápida y que, por tanto, no fueron escenario de habitación. Esta opción asume una función original para la estructura -normalmente, la de almacén y/o "silo"-, y un relleno intencionado posterior compuesto por desechos, cuando ya ha perdido su utilidad - desechos secundarios, en términos de Schiffer-. Son los conocidos como "basureros" (p.e Martín de la Cruz 1985: 186; Ruiz Fernández 1987; Arteaga y Cruz-Auñón 1999: 613; Cruz-Auñón y Arteaga 2001: 705; Román y Conlin 2001; Conlin y Gómez Saucedo 2003).

La tercera opción, la que aquí asumimos, sostiene que nos encontramos ante depósitos estructurados con una marcada significación simbólica (Márquez 2001; Márquez y Fernández 2002; Márquez 2003, 2004, 2006a, 2006b, e.p). Estaríamos, pues, ante procesos específicos de formación del registro arqueológico, que no consisten, ni en la paulatina formación de "niveles culturales", como sería de esperar en auténticas cabañas, ni en la deposición masiva y despreocupada de desechos por razones de higiene, como ocurre en los basureros modernos.

En este panorama, discutiremos a continuación la tesis que defiende la existencia de auténticas cabañas semisubterráneas en estos yacimientos del sur peninsular durante el IV-III milenio a.C., si bien podemos adelantar que no observamos diferencias en la formación de los depósitos arqueológicos de los llamados fondos de cabaña con respecto a los considerados silos, por lo que la distinción tradicional entre ambos tipos de estructuras, creemos, debe ponerse en entredicho.

\section{FONDOS DE CABAÑA Y FORMACIÓN DEL REGISTRO ARQUEOLÓGICO}

Las lecturas que reconocen en las cubetas excavadas en la roca los cimientos de cabañas-pozos han sido muy poco exigentes con la fenomenología arqueológica con la que estaban tratando. Un ejemplo claro está en la amplísima panoplia terminológica empleada, según qué autores, para denominarlas (fondos, sótanos, lugares de vivienda, estructuras circulares, grandes estructuras de cobijo, hitos habitacionales o productivos, cabañas semitrogloditas, estructuras de índole productiva, infraestructuras...), lo que es consecuencia de las debilidades de los argumentos empleados tanto para discriminarlas de otras estructuras como, simplemente, para describirlas. Son muchas y variadas las objeciones que se pueden hacer a esta hipótesis. Las más importantes afectan directamente a la formación del registro arqueológico en su interior y nos permiten afirmar que el relleno arqueológico de los denominados "fondos" no es primario, sino secundario (de nuevo, sensu Schiffer 1987), y de ninguna manera aleatorio.

En primer lugar, y en contra de lo que cabría esperar, llama poderosamente la atención que tras la ocupación prolongada de estas estructuras de hábitat o fondos de cabaña, que queda manifiesta, supuestamente, por la gran colmatación de sedimentos que se hallan en su interior, es casi imposible, en los numerosos casos estudiados en nuestra región, detectar evolución alguna en la cultura material a lo largo de toda la secuencia de una misma estructura, desde sus depósitos más profundos hasta los superiores ${ }^{2}$, lo que debería ocurrir si estuviéramos inequívocamente ante los depósitos correspondientes a auténticos "niveles culturales". Por otro lado, las tasas de deposición tanto de materiales arqueológicos como, sobre todo, de sedimentos, resultan, a todas luces, excesivas para ser producto de actividades domésticas cotidianas. Si la interpretación de los pozos como "fondos" fuese acertada, estaríamos ante cabañas con un escaso tiempo de uso (unos pocos años a lo sumo, según McGuire y Schiffer 1983), sin evidencias de mantenimiento o reparaciones estructurales, y con un relleno culturalmente uniforme. ¿Cómo se explicaría, entonces, que la deposición de detritus domésticos en un suelo de ocupación generase una extraordinaria potencia estratigráfica, a veces de hasta 2 metros, en tan poco tiempo?

En esta misma línea, cabe referir la ausencia de un patrón sedimentario en la formación de yacimientos de esta naturaleza, como, oportunamente, se ha apuntado para el caso de Papa Uvas (Martín de la Cruz y Lucena 2003: 159). No se encuentran depósitos arqueológicos en

2. Con cierta frecuencia se mencionan restos de época campaniforme en el depósito más superficial del relleno de algunos pozos, en contraste con el contenido artefactual de otros niveles del mismo. Pero ello no se debe, en nuestra opinión, a una evolución cultural a lo largo de la secuencia reflejo de una formación lenta. Por el contrario, se trata de una consecuencia bien conocida de la dinámica deposicional de pozos de este tipo (Hill 1995: 52): los depósitos, formados rápidamente, tienden a asentarse y comprimirse con el paso del tiempo debido sobre todo a la descomposición de restos orgánicos, dejando una depresión en superficie claramente visible por poblaciones posteriores. Estas poblaciones (en este caso, campaniformes) pueden, intencionadamente o no, rellenar esos hoyos o depresiones con materiales de su época, sin que ello suponga que tuvieron algo que ver en su construcción y su relleno original. 


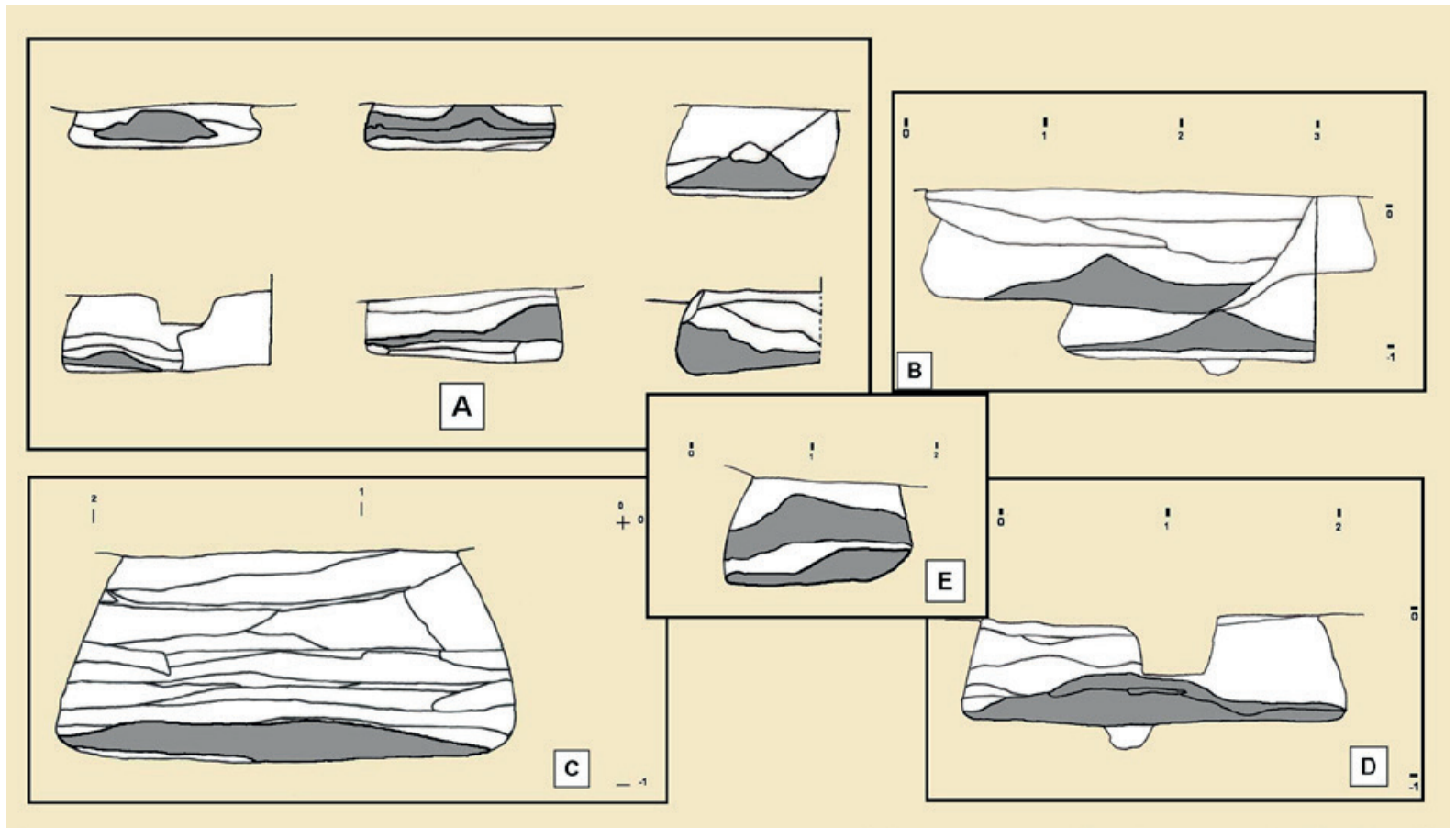

Figura 1. Acumulaciones sedimentarias anómalas en el relleno de los pozos de Polideportivo de Martos y Marroquíes Bajos. A) Estructuras para almacenaje de Polideportivo de Martos; elaboración propia a partir de Lizcano 1999: 89. B, C, y D) Complejo estructural 25 ("fondo"), Estructura 15 ("almacenaje" y Complejo estructural 17 ("fondo") de Polideportivo de Martos; elaboración propia a partir de Lizcano 1999: 111, 93 y 111, respectivamente. E) Estructura XV (“fondo") del sector UA-23 de Marroquíes Bajos; elaboración a partir de Burgos, Pérez y Lizcano 2001: fig. 5.

el interior de una estructura cuya formación pueda relacionarse claramente con la formación de niveles de otras estructuras cercanas, como serían, por ejemplo, niveles de incendio o inundación comunes, lo que, en principio, invalidaría la idea de que varias estructuras pudieran estar abiertas al mismo tiempo ${ }^{3}$. De ser realmente fondos de cabaña, nunca habría dos cabañas en uso a la vez.

Otro de los evidentes puntos débiles de la hipótesis de los fondos de cabaña es la existencia de depósitos (supuestos niveles culturales) que presentan, de unos sectores a otros de su extensión, grandes diferencias en su grosor, contingencia que se documenta tanto en los pozos considerados fondos de cabaña como en las

3. En el yacimiento de Polideportivo de Martos, se ha planteado una única conexión estratigráfica directa en un hipotético nivel de inundación común a varios pozos (Lizcano 1999:121-122), aunque en nuestra opinión la simple semejanza morfológica de algunos depósitos no constituye fundamento suficiente para mantener esta hipótesis, especialmente teniendo en cuenta el contexto en el que supuestamente tiene lugar-que como veremos, parece ser el de estructuras colmatadas intencionadamente-. El hecho de que en ningún yacimiento de esta naturaleza conocido en nuestra región se haya documentado nada similar nos obliga a dejar en suspenso esa posibilidad hasta que se realicen nuevos estudios. supuestas estructuras de almacenaje o silos. Estas diferencias, además de traducirse en sectores donde el estrato se adelgaza hasta desaparecer, se manifiestan, también, bajo la forma de cúmulos o amontonamientos de sedimentos que en ocasiones adoptan incluso una clara forma cónica (fig. 1 y 2). Se ha intentado explicar esta extraña característica del relleno en algunos de los fondos de cabaña. Por ejemplo, en el yacimiento de Carmona se explica como "un derrumbe o algún elemento de apoyo" (Román y Conlin 2001: 530). En Martos, por el contrario, se lo considera una consecuencia de la acumulación de sedimentos sobre un refuerzo para entibar el poste de la cabaña u hogar central (Lizcano 1999: 85; Lizcano y Cámara 2004: 231). Pero, como se observa en la fig. 1, si bien los amontonamientos son un fenómeno que a veces -sólo a veces- aparece asociado a las consideradas "cubetas de poste central", en otros casos la acumulación se da en un lateral y no en el centro del pozo. Además, no tiene sentido esta vinculación con supuestos hoyos de poste en los casos en los que el cúmulo sedimentario no se asienta directamente sobre el fondo de la estructura, sino sobre otros estratos previamente depositados que no presentan esta anomalía. 

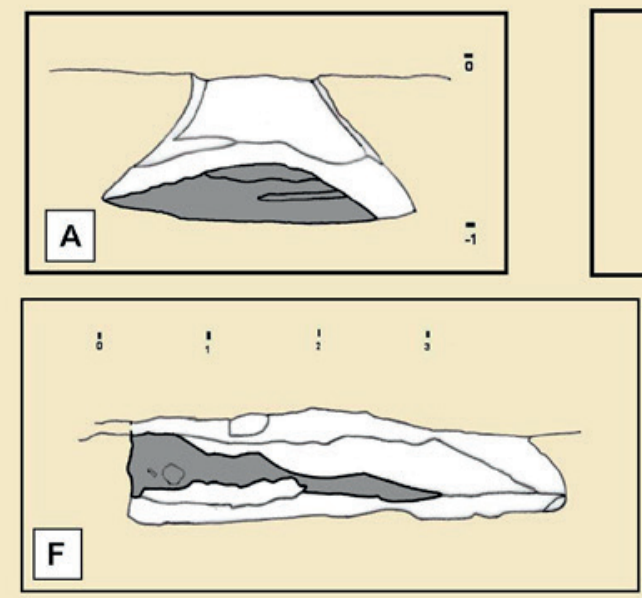
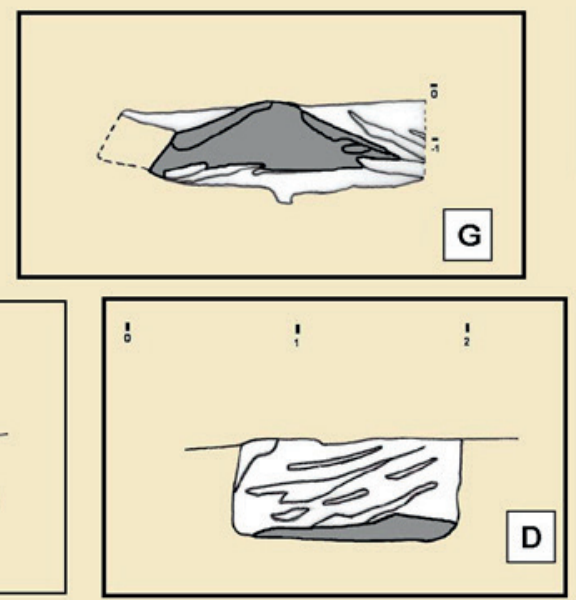
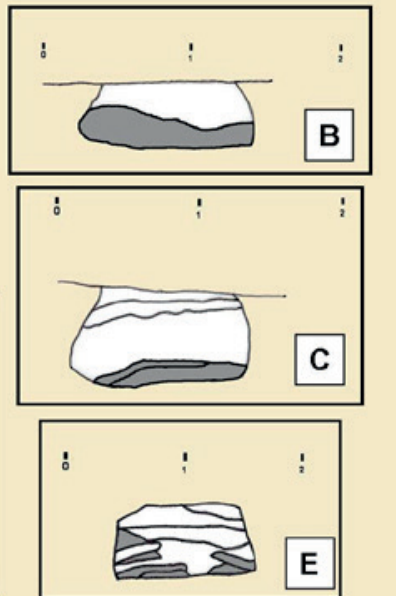

Figura 2. Acumulaciones sedimentarias anómalas en el relleno de los pozos de Papa Uvas y otros yacimientos. A) Estructura 1 del cuadro C, Papa Uvas: elaboración propia a partir de Martín de la Cruz y Lucena 2003: fig. 7. B, C, D y F) Fondo 8 del Sector A (corte A-8.1), Fondo 7 (corte A-7.3), Fondo 10 del sector C (corte D-3.1) y Fondo 3 del corte C-4.3 del Sector B, Papa Uvas; elaboración propia a partir de Martín de la Cruz 1986: fig. 29, fig. 28, fig. 106 y fig. 62, respectivamente. E) Estructura 4 del corte F.-2.2 de Papa Uvas (Campaña 1979); elaboración propia a partir de Martín de la Cruz 1985: fig. 18. G) Covacha I (“cabaña") de Pocito Chico; interpretación propia a partir de Ruiz Gil y López Amador 2001: Lam. 6.

Muy al contrario, creemos que la tendencia cónica de estos estratos -efecto de "reloj de arena"- se explica mejor como resultado de la práctica de arrojar cenitalmente sus componentes, probablemente desde el exterior del pozo, explicación ya apuntada en Papa Uvas (Martín de la Cruz y Lucena 2003: 153).

Otras disposiciones "anómalas", que igualmente aparecen en las estructuras en "negativo" -ya sean consideradas silo o fondos-, son aquellas en las que los estratos no se ordenan espacialmente de forma horizontal, sino con buzamientos muy notables, cuando no con una evidente tendencia a la verticalidad (fig. 3). No conocemos ninguna actividad "doméstica" que pudiera llevar a la formación de este tipo de depósitos. Es más, ¿cómo pudieron habitarse estas estructuras con semejantes acumulaciones de residuos restringiendo y entorpeciendo cualquier actividad, desde las económicas hasta las de mero descanso? Por el contrario, la deposición deliberada de sedimentos, artefactos y ecofactos, por ejemplo, dentro de un fardo o saco tendría muy probablemente un reflejo arqueológico muy similar al observado en algunas de las estratigrafías que venimos analizando -tendencia a la verticalidad-. Otros estratos con gran buzamiento parecen haberse formado "empujando" amontonamientos de sedimentos desde la periferia exterior de la estructura hasta hacerlos caer. Una última "anomalía" en el registro es la formación de grandes cúmulos de restos que en un suelo de ocupación normalmente aparecerían mucho más dispersos $o$ en menor densidad. Nos referimos a la documentación de montones de cenizas de hasta $30 \mathrm{~cm}$. de espesor (Lizcano 1999: 86), o de malacofauna (Martín de la Cruz y Lucena 2003: fig. 7).

Por último, cabe apuntar las dificultades que encuentra la tesis de los fondos de cabaña para explicar la presencia, en la formación de sus depósitos, de gran cantidad de objetos con carácter "especial", lo que nos permite vincular la fenomenología que estamos tratando con toda una tradición de deposiciones estructuradas a escala europea. Esta cuestión ha sido abordada en los últimos años (Márquez 2001; Márquez y Fernández 2002; Márquez 2003, 2004, 2006a, 2006b, 2007), por lo que aquí sólo efectuaremos una breve enumeración de los mismos: restos humanos, tanto completos y articulados como incompletos, siempre sin ajuar asociado; restos de animales (suidos, cánidos, bóvidos, cérvidos, ovicápridos y ocasionalmente otros como los équidos), a veces completos y articulados; piedras de pequeño y mediano tamaño; molinos fragmentados; piedras de gran tamaño colocadas en una posición central dentro del fondo de la estructura en negativo. En definitiva, toda una serie de artefactos y ecofactos cuya inserción en un espacio teóricamente doméstico resulta anómala. 


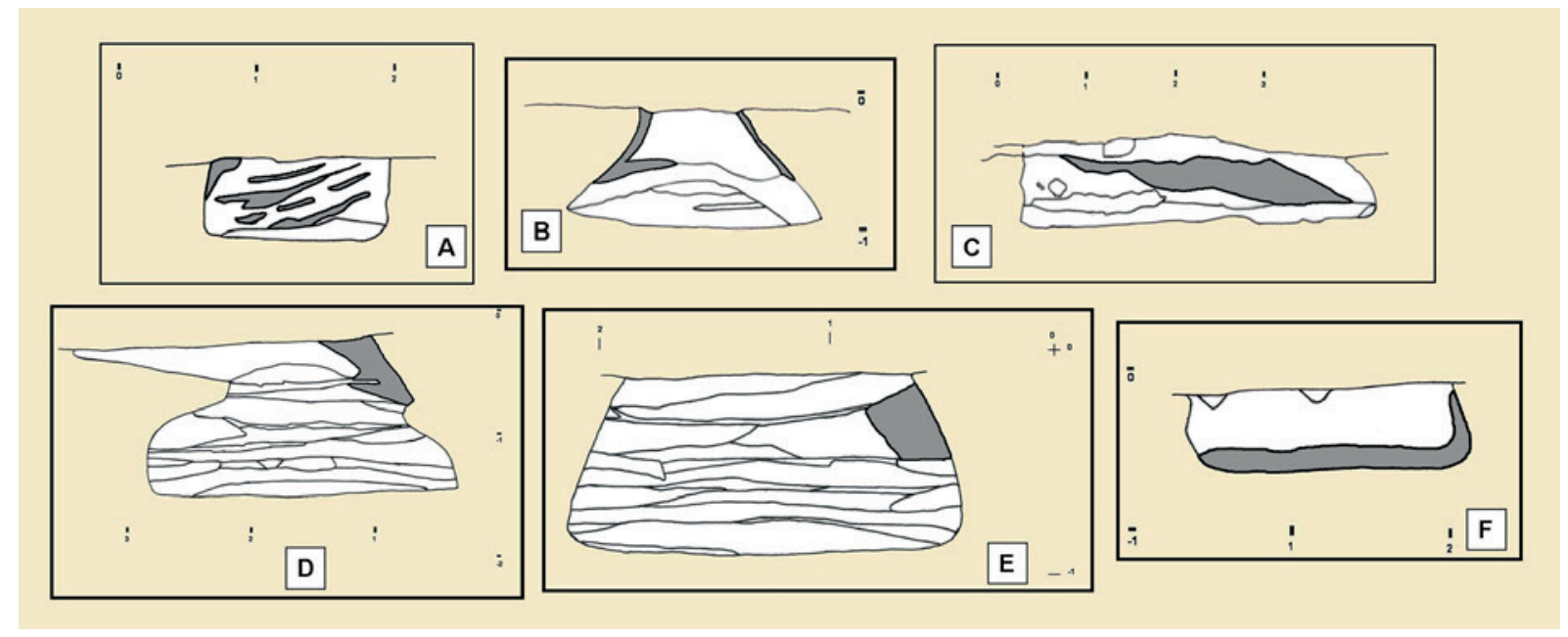

Figura 3. Depósitos verticales o con gran buzamiento. A) Fondo 10 del sector C (corte D-3.1), Papa Uvas; elaboración propia a partir de Martín de la Cruz 1986: fig. 106. B) Estructura 1 del cuadro C, Papa Uvas: elaboración propia a partir de Martín de la Cruz y Lucena 2003: fig. 7. C) Fondo 3 del corte C-4.3 del Sector B, Papa Uvas; elaboración propia a partir de Martín de la Cruz 1986: fig. 62. D y E) Complejo Estructural 12 ("fondo") y Estructura 15 ("almacenaje") de Polideportivo de Martos; elaboración propia a partir de Lizcano 1999: 108 y 93, respectivamente. F) "Silo" 19 del Cerro de San Cristóbal; elaboración propia a partir de Fresneda et. al. 1993: fig. 2.

\section{EL CONCEPTO DE CASA-POZO}

Al margen de la problemática de la formación del registro, la tesis de los pozos como cabañas semisubterráneas tiene otro punto débil: se ha formulado sin efectuar la pertinente definición del concepto de casa semisubterránea y su aplicación crítica al problema arqueológico que nos ocupa. Creemos que este error es crucial, hasta el punto de que si ello no fuera así probablemente la interpretación de las supuestas casas-pozo en el Neolítico y Calcolítico peninsulares se habría descartado hace tiempo. Este tema ha sido tratado en otro lugar más extensamente (Jiménez Jáimez, e.p.), por lo que en este momento, simplemente, apuntaremos algunas de las ideas allí desarrolladas.

Las casas-pozo o casas subterráneas o semisubterráneas, denominadas en el mundo anglosajón pithouses o dwelling-pits, son chozas construidas tomando como base una fosa excavada en el suelo cuyos muros son las paredes interiores de dicha fosa, a las que con frecuencia hay que añadir algún tipo de pared "exenta" o "aérea" con materiales perecederos o bloques de piedra que se superpone a las primeras. Resulta en ocasiones realmente complicado distinguir arqueológicamente las casas semisubterráneas de otras estructuras en negativo de función y significado diverso. De hecho, la polémica a este respecto no sólo ha tenido lugar en la Península Ibérica, pues desde hace décadas se vienen discutiendo problemas similares en regiones como la Península Balcánica (Buttler 1936; Chapman 2000), entre otras.

No obstante, abundantes estudios etnográficos y de arqueología experimental (p.e. Buttler 1936; McGuire y Schiffer 1983; Gilman 1987; Chapman 2000: 87) arrojan luz sobre este asunto al mostrarnos que, independientemente del contexto en el que se inserten, las casas-pozo como espacios arquitectónicamente creados para residir en ellos exigen ciertas características básicas que permitan unas elementales condiciones de habitabilidad. Ante todo, se ha puesto de manifiesto la necesidad ineludible de disponer mecanismos para evitar la entrada de agua, insectos y otros agentes desagradables o perjudiciales para la vida cotidiana de sus habitantes. Junto a ello, las particularidades del acceso al espacio doméstico, bien de forma cenital, bien a través de un pasillo o corredor, y la necesidad de dejar un vano o agujero en el techo para la evacuación del humo del hogar, configuran sistemas de cubrición más complejos de lo que en principio cabría esperar y que conocemos para las estructuras superficiales. Por todo ello, resultan erróneamente simplificadoras las propuestas de techumbres poco complejas como las de aspecto cónico con un único poste central que dominan las explicaciones prehistóricas en nuestro ámbito geográfico (Lizcano et al. 1991-1992; Lizcano 1999; Ruiz Gil y López Amador 2001: 65). 


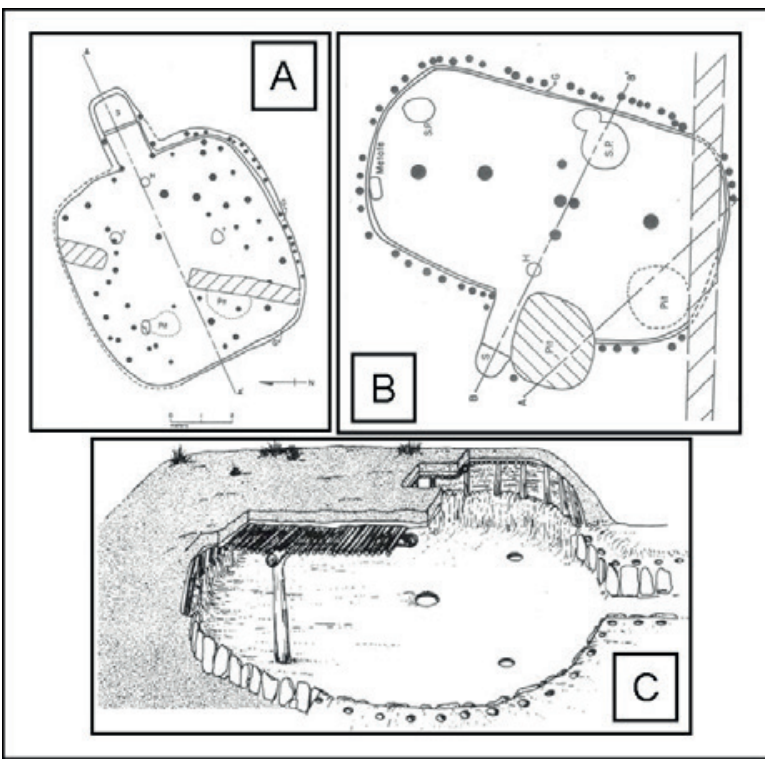

Figura 4. Ay B) Plantas de dos pithouses en el poblado hohokam de Snaketown, Arizona, Estados Unidos (Seymour y Schiffer 1987: fig. 12.2 y fig. 12.4 respectivamente). C) Reconstrucción ideal de un pithouse basketmaker, según Charles M. Carrillo (reproducido en Cordell 1984: fig. 3.4, B).

Concretamente, los requerimientos técnicos específicos de esta forma arquitectónica se materializan en tres características casi imprescindibles para los ejemplos arqueológica y etnográficamente documentados, a saber, planta regular, unas paredes de la fosa base aproximadamente rectas y la colocación de una serie continuada de postes en la periferia exterior del pozo (fig. 4). La única solución tecnológica que no conlleva la presencia de abundantes postes de madera exteriores para crear una cubierta compleja consiste en levantar un muro de escasa altura sobre el suelo, en la periferia del pozo, y apoyar sobre él, y no sobre el suelo, una serie de vigas horizontales que sostendrían el tejado, técnica de la cual poseemos algunas, muy escasas, noticias etnográficas (Buttler 1936: fig. 9).

En suma, pensamos que la idea de la cabaña semisubterránea no puede ser empleada para forzar el registro arqueológico de la Península Ibérica y adecuarlo a nuestras ideas preconcebidas sobre la existencia de poblados en el Neolítico y Calcolítico sin someterla a la correspondiente crítica. En los apartados siguientes, esperamos demostrar que el concepto de casa-pozo no es aplicable al ámbito de estudio referido, más allá de los problemas de la formación del registro. Como veremos, la inexistencia de hoyos de poste exteriores (y la dudosa naturaleza de los interiores), la irregularidad de las paredes, las insuficiencias del espacio interior en cuanto a sus dimensiones, los problemas del sustrato de base y otros elementos son manifiestamente inadecuados y desaconsejan seguir considerando esta fenomenología arqueológica como cabañas semisubterráneas.

\section{LOS ASPECTOS ARQUITECTÓNICOS \\ DEL PROBLEMA (I): LA CREACIÓN Y EL AISLAMIENTO DEL ESPACIO DOMÉSTICO}

No puede olvidarse que la inmensa mayoría de los llamados poblados de fosos se encuentran excavados en el mismo tipo de roca, a saber, calizas margas, contingencia determinante a la hora de evaluar su conveniencia como base para la excavación de casas-pozos.

Determinante, por ejemplo, en la base naval de Rota (Ruiz Fernández y Ruiz Gil 1989: 8), donde se comprobó experimentalmente que las margas que constituían el sustrato geológico en el que se habían excavado las estructuras poseían una capacidad enorme para la absorción hídrica, lo que descartaba un hipotético uso de las mismas como contenedores de agua. Esta propiedad también se ha subrayado para el sustrato geológico del yacimiento de Los Pozos, en Higuera de Arjona (Hornos et. al. 1987: 201), así como en las margas del Aljarafe sevillano, en cuyo seno se encuentran las estructuras en negativo de Valencina de la Concepción, que poseen igualmente un alto grado de permeabilidad. Lo relevante para nuestra discusión es comprobar la tendencia natural de esta roca a filtrar el agua y posteriormente retener su humedad, lo que tiene importantes implicaciones funcionales no discutidas cuando se defiende el carácter habitacional de estas estructuras, en el sentido de que sería un inconveniente nada despreciable a la hora de mantener el espacio interno habitable. Puesto que la roca madre permite los desplazamientos de agua en vertical, en las estructuras de sección acampanada -la gran mayoría de las que estamos analizando- ya no sería necesaria la entrada de agua directamente al interior del pozo para provocar problemas a sus habitantes, sino que cualquier cantidad abundante de agua que cayera sobre la roca podría filtrarse y humedecer las paredes interiores del pozo, tardando bastante tiempo en secarse.

A todo lo dicho sobre la permeabilidad de las margas, cabe añadir que, como se ha apuntado (Fernández Gómez y Oliva 1980: 23), la cantidad de agua contenida afecta a su dureza, convirtiéndolas en rocas muy blandas. Esto significaría que, además, las paredes estarían en peligro de derrumbarse al ablandarse la roca en la que están labradas. 
Pero la despreocupación de los constructores prehistóricos de casas-pozo parece no tener límites, pues muchas de las estructuras -fondos o silos- se excavan directamente sobre estructuras más antiguas, de manera que parte de los nuevos fondos o paredes no están labrados en la roca madre, sino en el relleno de una estructura previamente excavada y colmatada -en otras palabras, excavados en tierras oscuras, generalmente no muy compactas y con gran densidad de objetos y restos humanos y animales-. Los casos conocidos se cuentan por decenas, pero como ejemplo podemos tomar un "fondo" de Polideportivo de Martos (fig. 1. B). Todo ello no sólo hace más difícil la tarea de mantener el hipotético espacio doméstico alejado de la humedad, sino que incide en la gran debilidad que tendrían las paredes así construidas, que tenderían a derrumbarse con gran facilidad y en las que sería muy complicado colocar postes de sustentación, muretes periféricos o sistemas para sellar el interior.

La naturaleza arquitectónica de estas supuestas cabañas semisubterráneas obligaría a adoptar soluciones técnicas para su cierre. Por lo que conocemos etnográficamente una posible solución sería la elevación de un muro periférico en el exterior de la estructura, al nivel del suelo, sobre el que se apoyarían vigas horizontales, creando una superficie plana en la que, necesariamente, se debería ubicar un agujero para el acceso cenital. Esta posibilidad presenta graves inconvenientes para ser conciliada con el registro arqueológico que conocemos en nuestra región. Por ejemplo, no tenemos noticias de la aparición de grandes cantidades de adobe en el relleno y/o alrededores de las casas-pozo, lo que debería ser una constante evidencia arqueológica como resultado tanto de la caída del muro como del recubrimiento del tejado. De ser cierta esta hipótesis pensamos que se generarían auténticas monteras de barro en, y sobre, las estructuras excavadas.

Es cierto que en ocasiones se apunta la aparición de restos de adobe, que mecánicamente se relacionan, por mimetismo, con el sistema de construcción, pero su presencia cualitativa no nos basta para argumentar una técnica arquitectónica que se supone generalizada en cientos de "cabañas" prehistóricas. Sólo podemos estar seguros de su uso para la construcción cuando el adobe presenta improntas vegetales y cuando podemos relacionar esos hallazgos con nuestros pozos (Martín de la Cruz 1986: 23; Cabrero 1987: 182; Murillo et. al. 1987 : 313; Santana 1993: 550; Lizcano 1999: 85; Román y Conlin 2001: 532) y no con niveles de épocas posteriores, que son los que la mayor parte de las veces presentan tales restos. Más si cabe, si tenemos en cuenta lo fácil que es confundir ese material con fragmentos de otros artefactos de barro poco cocido como los llamados "morillos", crecientes, o ídolos que se nos muestran, cada día más, como una constante en el contenido material documentado en el relleno de estos pozos (Lucena 2004: 233-236). Por otro lado, a partir de la naturaleza del relleno arqueológico de los pozos es muy arriesgado sostener que los adobes con improntas se encuentran en posición primaria y que corresponden a restos de un hipotético sistema de cubrición del propio pozo: más bien parecen haber sido arrojados al fondo desde el exterior junto con el resto de los artefactos (serían, pues, desechos secundarios).

Otra técnica que permitiría el cierre externo de la casa-pozo, imprescindible, como ya hemos comentado, a tenor de las evidencias etnográficas, sería la colocación de postes en la periferia exterior del pozo. Esta situación debería dejar algunas evidencias arqueológicas muy claras, en tanto que no bastaría un hoyo de poste aislado en el exterior, sino que sería necesaria una serie bien distribuida de hoyos de postes, circunstancia desconocida en el ámbito de estudio que estamos abordando. Debemos hacer referencia aquí a la noticia de la excavación de una estructura en El Negrón (Rivero et al. 1989; Cruz-Auñón y Rivero 1990), donde se habla de hoyos de poste interiores, pero no exteriores al propio pozo. Por otro lado, la hipótesis de un solo poste interior -casi un axioma en las explicaciones al uso-, tiene muy poca consistencia etnográfica y resulta inconsistente si la pretendemos conciliar con el registro arqueológico. En el próximo epígrafe analizaremos estas propuestas con más detalle.

Para finalizar, hay que añadir que los sistemas de acceso no han sido explicados con detalle por quienes defienden la tesis de los fondos de cabañas. En este sentido, llama la atención que, a pesar de la profundidad notable de muchos de los fondos, no existan escalones, rampas o pasillos que comuniquen el interior con el exterior. Los escasos escalonamientos propuestos (Cabrero 1990; Murillo 1991: 559) creemos que son resultado, simplemente, del perfil generado por la reexcavación de unos pozos sobre otros previamente construidos y clausurados.

\section{LOS ASPECTOS ARQUITECTÓNICOS DEL PROBLEMA (II): LA ORDENACIÓN DEL ESPACIO INTERIOR}

Centrándonos en las características internas de estos "hábitats", extraña, en primer lugar, que, comparativamente, las dimensiones habituales de los "fondos de cabaña" de la Prehistoria andaluza son bastante más 
reducidas que la mayoría de los casos de habitaciones semisubterráneas etnográficamente conocidas, pues, salvo casos excepcionales, no suelen superar los 2,5 metros de diámetro en su fondo. Debe tenerse en cuenta además que su perfil, en numerosas ocasiones acampanado, reduce notablemente las dimensiones reales de la estructura, más allá del diámetro de su fondo. El hecho de que la boca pueda llegar a ser mucho menor que el fondo de los pozos condiciona enormemente la habitabilidad del, así considerado, espacio doméstico, de manera que en la mayoría de las estructuras clasificadas como fondos de cabaña existe una gran fracción del espacio en la que sus ocupantes no podrían mantenerse de pie. Si bien es cierto que para dormir o tener refugio no es imprescindible disponer de espacio para levantarse y andar erguido, sí creemos que la realización de las actividades económicas cuyos residuos, siguiendo la tesis de los fondos de cabaña, rellenarían el pozo, sería casi imposible. Solo quedaría como área de actividad verosímil el cilindro imaginario que estaría marcado por la proyección de la boca del pozo en su fondo, espacio todavía más reducido si, como con frecuencia se afirma, hubiese postes centrales de sustentación de la techumbre u hogares delimitados (Lizcano 1999, entre otros). Además, la forma de las paredes, en muchas ocasiones con una marcada forma de campana, no se acerca a los ejemplos etnográficos y arqueológicos conocidos y a las informaciones procedentes de la arqueología experimental en relación con su aislamiento frente a agentes externos desagradables (ver más arriba).

A las reducidas dimensiones de la mayoría de "fondos" hay que añadir que, según esta tesis, la hipotética ocupación sistemática y permanente del pozo generaría una acumulación paulatina de desechos que iría elevando paulatinamente el nivel del suelo de ocupación, y reduciendo, se supone, el espacio habitable, hasta la colmatación total o casi total de aquél; hecho que, en ocasiones, obligaría incluso a reexcavar el substrato geológico para ganar espacio (Lizcano 1999: 104 y 110). Tampoco queda clara la relación entre la altura inicial de la techumbre, que se supone una constante, y la variable de los suelos de ocupación que cambian, según estos argumentos, a lo largo de la vida útil de la cabaña.

Ya hemos adelantado que la mayoría de autores asumen la existencia de un poste interior y central sobre el que se sostiene la cubierta cónica de la casa semisubterránea. De manera general se ha señalado la presencia de hoyos de poste en Polideportivo de Martos (Lizcano et. al. 1991-1992; Lizcano 1999: 85) y en la "covacha 1" de Pocito Chico (Ruiz Gil y López Amador 2001: 65), además de algunas evidencias de difícil interpretación en Papa Uvas - prácticamente descartadas por su propio excavador- (Martín de la Cruz 1985: 31-32 y 153), y el El Negrón (Rivero et al. 1989; Cruz-Auñón y Rivero 1990).

Creemos que las pequeñas cubetas que se documentan en el fondo de algunas estructuras (Lizcano et. al. 1991-1992; Lizcano 1999; Ruiz Gil y López Amador 2001: 65) interpretadas como hoyos de poste centrales, no pueden ser hoyos de poste por varias razones, al menos no dentro de una explicación más general que considera esos pozos como chozas semisubterráneas:

1) No se observa una discontinuidad estratigráfica, ni en planta ni en perfil, donde hipotéticamente estaba situado el poste. Dicha discontinuidad sería evidente si, como se pretende, la estructura se fue colmatando de sedimentos alrededor del poste y más tarde éste se descompuso.

2) No se perciben indicios de reparación ni de colocación del poste en otros puntos de la secuencia, algo que sería obligado porque: a) el poste no tardaría mucho en deteriorarse al estar hincado directamente sobre el suelo (McGuire y Schiffer 1983: 291); b) el nivel del suelo de habitación iría elevándose y sería imprescindible subir la altura de la techumbre para que los ocupantes no se viesen obligados a andar permanentemente agachados.

3) Las pequeñas cubetas de poste aparecen también en estructuras que de ninguna manera, por sus dimensiones y por su forma, podrían ser cabañas, como por ejemplo la XLII del Polideportivo de Martos (Lizcano 1999: 89).

4) El poste central disminuiría, aún más, el ya de por sí pequeño espacio en el interior de los pozos y en el cual, según se cree, se realizaban las actividades económicas cuyos desechos se acumulaban con gran rapidez.

5) Al hilo de lo comentado más arriba, aceptar la presencia de un único poste central sería asumir, además, que el acceso al interior se pudiera realizar a través de la techumbre, es decir, sin corredor o escalera de entrada a la choza -algo impensable en un sistema de cubrición tan simple como el que se propone-, y, al mismo tiempo, negar la colocación de un sistema que permitiera precintar y anclar la parte vegetal de la cubierta al suelo.

Quizá por ello las propuestas iniciales (Lizcano et. al. 1991-1992; Lizcano 1999) han sido matizadas posteriormente, afirmándose sobre estos hoyos que pueden ser "hoyos de poste/escalera de acceso" (Lizcano y Cámara 2004: 231).

En cuanto al hallazgo de hipotéticos hogares en el interior de estos fondos, lo común es que se denomine 
hogar a simples acumulaciones de cenizas, si bien algunas excavaciones han localizado círculos de barro o piedras que podrían estar delimitando hogares (Cruz-Auñón y Rivero 1990: 278; Santana 1993: 548; Lizcano 1999, en varias estructuras), y a veces incluso se habla de hogares enclavados en rehundimientos o huecos (Cabrero 1987: 182; Conlin y Gómez Saucedo 2003: 1260). En realidad, ni la presencia de restos de combustión ni la de un hogar propiamente dicho debe llevarnos automáticamente a pensar en actividades de cocina o en un recurso para calentar la hipotética vivienda. Hay muchas razones por las que una sociedad prehistórica puede encender un fuego. El propio concepto de basurero puede implicar la incorporación de grandes cantidades de cenizas al interior de los pozos o la incineración deliberada de residuos domésticos dentro de las propias estructuras en negativo; si contemplamos la colmatación de los pozos como un acto simbólico, las posibilidades aumentan más aún. Así pues, una hoguera no es suficiente para definir por sí sola una vivienda.

\section{POZOS Y POBLADOS EN EL NEOLÍTICO Y CALCOLÍTICO PENINSULARES}

De lo expuesto en los apartados precedentes creemos deducir que las evidencias arqueológicas que hasta ahora han sido interpretadas como casas-pozo neolíticas y calcolíticas en el sur peninsular son poco consistentes. Y no lo son por dos hechos fundamentales: a) el material sedimentario y artefactual que las rellena no se corresponde con una superposición de "niveles culturales" o "suelos de ocupación" formados sucesivamente a lo largo del tiempo, sobre todo si tenemos en cuenta que, de ser cabañas, la vida útil de estas estructuras sería muy breve; muy al contrario, todo parece indicar un relleno rápido e intencionado (secundario), todo ello sin necesidad de entrar a valorar las características "especiales" de muchos de los contenidos (cadáveres animales y humanos, por ejemplo); y b) técnica y físicamente no cumplen con unos requisitos mínimos de habitabilidad, por dimensiones, forma del alzado, acondicionamiento interno, ausencia de sistemas de cubrición, etc.

De ser correctas nuestras críticas, las consecuencias que se derivan de ellas nos enfrentan a lo que se han convertido en tres problemas centrales de la Prehistoria del sur de la Península Ibérica (y claro está, de otros muchos espacios europeos donde proliferan estos yacimientos):

1) Hay que replantear, como ya ha ocurrido en otras regiones continentales, la metodología de estudio, el análisis y la representación de estas estructuras.
Tenemos que superar prejuicios que nos condenan a tener que demostrar que estas estructuras en negativo no son silos o fondos de cabaña; lo que habría que argumentar es precisamente lo contrario, su supuesta función doméstica. Ante todo debemos reconocer que estamos ante unos complejísimos procesos de formación del registro arqueológico. Evidentemente la tarea no es fácil. Estamos convencidos de que el relleno de la mayoría de estos pozos es resultado de una deposición normalizada o estructurada (Márquez 2001; Márquez y Fernández 2002). Pero tal hipótesis de trabajo no resuelve muchas de las dudas que generan la formación del registro arqueológico en los yacimientos de fosos. No obstante, creemos honestamente que hay que retomar la discusión desde el punto en el que nos encontramos (con más sombras que luces) y no adoptar posturas que descansen sobre cómodos prejuicios interpretativos y carencias en la información procedente de las memorias arqueológicas.

2) La segunda consecuencia que extraemos, desarrollada con profundidad en otro lugar (Márquez 2006b), es la dificultad que entraña identificar, en los yacimientos de fosos, tanto los primeros poblados humanos como los más precoces centros de poder existentes en Prehistoria Reciente del sur de la Península, ya desde finales del IV milenio. No se puede hacer descansar unos modelos históricos de tal envergadura sobre un registro arqueológico tan ambiguo. Es un lugar común, en la discusión actual sobre esta problemática en toda Europa, que los recintos de fosos desafían la idea tradicional del asentamiento o poblado. Es más, estamos convencidos de que el concepto o categoría "poblado" ha perdido su valor descriptivo o analítico para definir estos yacimientos, lo que subraya importantes limitaciones instrumentales de nuestro método. Esto requiere un compromiso de todos para aunar esfuerzos por presentar modelos alternativos que no necesariamente tienen que ser ajenos al proceso de aparición de la desigualdad social, pero que deben caracterizarse por una mayor correspondencia con el registro arqueológico disponible.

3) En cuanto a las estructuras en negativo en sí mismas, con respecto a su función y significado, una vez señalado el carácter secundario, deliberado y secuenciado que creemos observar en su colmatación, cabe preguntarse por la naturaleza de los materiales arrojados a su interior, por el significado del propio acto de la deposición, y sobre todo, por las prácticas sociales que llevaron a la formación de tan peculiares registros arqueológicos. Sin olvidar, por supuesto, que, antes de su clausura definitiva, los pozos pudieron o no cumplir otras funciones y significados que habrá que intentar desentrañar. 


\section{BIBLIOGRAFÍA.}

ARTEAGA, O. y CRUZ-AUÑÓN (1999): “Una valoración del Patrimonio Histórico en el Campo de Silos de la Finca El Cuervo-RTVA. (Valencina de la Concepción, Sevilla) Excavación de urgencia de 1995”, Anuario Arqueológico de Andalucía 1995, vol. III, actividades de urgencia: 608-616.

BURGOS, A.; PÉREZ, C. y LIZCANO, R. (2001): "Actuación arqueológica realizada en la piscina comunitaria de los bloques A1, A2, A3, A6, A7 y A8 del sector UA-23 de Marroquíes Bajos de Jaén", Anuario Arqueológico de Andalucía 1998, III, vol. 1: 402-413.

BUTTLER, W. (1936): "Pits and Pit-dwellings in Southeast Europe", Antiquity 10: 25-36.

CABRERO, R. (1987): "Informe Preliminar Sobre las Excavaciones Arqueológicas Realizadas en el Yacimiento de Amarguillo II (los Molares, Sevi1la)", Anuario Arqueológico de Andalucía 1986, II: 180-185.

CABRERO, R. (1990): “El Poblado de la Edad del Cobre Denominado Amarguillo II (los Molares, Sevi1la). Informe Preliminar tras la Excavación Sistemática de 1987', Anuario Arqueológico de Andalucía 1987, II: 276-277.

CHAPMAN, J. (2000): "Pit-digging and Structured Deposition in the Neolithic and Copper Age", Proceedings of the Prehistoric Society 66: 61-87.

CONLIN, E. y GÓMEZ SAUCEDO, Mª T. (2003): "Excavaciones arqueológicas de urgencia en la calle Dolores Quintanilla, no 12. Carmona (Sevilla)", Anuario Arqueológico de Andalucía 2000, vol. III (2): 1257-1265.

CORDELL, L. (1984): Prehistory of the Southwest. Academic Press.

CRUZ-AUÑÓN, R. y ARTEAGA, O. (1999): “Acerca de un campo de silos y un foso de cierre prehistóricos ubicados en la Estacada Larga (Valencina de la Concepción, Sevilla). Excavación de urgencia de 1995”, Anuario Arqueológico de Andalucía 1995, III, Actuaciones de urgencia: 600-607.

CRUZ-AUÑÓN, R.; MORENO, E.; CÁCERES, P. y VALVERDE, Ma . (1995): "Informe provisional de la excavación sistemática en el yacimiento de El Negrón (Gilena, Sevilla). Campaña de 1991", Anuario Arqueológico de Andalucía 1992, II, Actividades Sistemáticas: 347-351.

CRUZ-AUÑÓN, R.; RIVERO, E. (1990): "Yacimientos del Negrón (Gilena, Sevilla), campaña 1987". Anuario Arqueológico de Andalucía 1987, II, Actividades Sistemáticas: 278-280.
FERNÁNDEZ GÓMEZ, F. y OLIVA, D. (1980): “Los ídolos calcolíticos del Cerro de la Cabeza (Valencina de la Concepción, Sevilla)", Madrider Mitteilungen 21: 20-44.

FRESNEDA, E.; RODRÍGUEZ ARIZA, Mª O.; LÓPEZ LÓPEZ, M. y PEÑA, J. M. (1993): "Excavación de urgencia en el Cerro de San Cristóbal (Ogijares, Granada). Campaña de 1991”, Anuario Arqueológico de Andalucía 1991, III: 214-220.

GILMAN, P. A. (1987): "Architecture as Artifacts: Pit Structures and Pueblos in the American Southwest", American Antiquity 52, $\mathrm{n}^{\circ}$ 3: 538-564.

HILL, J. D. (1995): Ritual and Rubbish in the Iron Age of Wessex. A study on the formation of a specific archaeological record. BAR British Series, 242.

HORNOS, F.; NOCETE, F. y PÉREZ, C. (1987): “Actuación arqueológica de urgencia en el yacimiento de los pozos en Higuera de Arjona (Jaén)", Anuario Arqueológico de Andalucía 1986, III, Actividades de Urgencia: 198-202.

JIMÉNEZ JÁIMEZ, V. (e.p.): “Pithouses versus pits. Algunos apuntes para la resolución de un problema arqueológico".

LIZCANO, R. (1999): El Polideportivo de Martos (Jaén): un yacimiento neolítico del IV Milenio AC. Nuevos datos para la reconstrucción del Proceso Histórico del Alto Guadalquivir. Córdoba.

LIZCANO, R.; CÁMARA, J.A.; RIQUELME, J.A.; CAÑABATE, Ma . L.; SÁNCHEZ, A. y AFONSO, J.A. (1991-1992): "El polideportivo de Martos. Producción económica y símbolos de cohesión en un asentamiento del Neolítico final en las campiñas del Alto Guadalquivir", Cuadernos de Prehistoria de Granada 16-17: 5-101.

LIZCANO, R. y CÁMARA, J. A. (2004); "Producción económica y sedentarización. El registro arqueológico del Polideportivo de Martos (Jaén)", en Sociedades recolectoras y primeros productores. Actas de las Jornadas temáticas andaluzas de arqueología: 229-248. Junta de Andalucía, Sevilla.

LUCENA, A. (2004): "Estructuras y contenidos cerámicos en el yacimiento arqueológico de Papa Uvas (Aljaraque, Huelva): campaña de 1994", II y III Simposios de Prehistoria Cueva de Nerja: 227-237.

MÁRQUEZ, J. E. (2001): "De los campos de silos a los agujeros negros': sobre pozos, depósitos y zanjas en la Prehistoria Reciente del Sur de la Península Ibérica", Spal 10: 207-220. http://dx.doi. org/10.12795/spal.2001.i10.14

MÁRQUEZ, J. E. (2003): "Recintos Prehistóricos Atrincherados (RPA) en Andalucía (España): Una propuesta interpretativa.", en Oliveira Jorge, S (coord), 
Recintos murados da Pré-história recente. Técnicas construtivas e organizaçao do espaçio. Conservaçao, restauro e valorizaçao patrimonial de arquitecturas pré-históricas: 269-284. Oporto-Coimbra.

MÁRQUEZ, J. E. (2004): "Muerte ubícua: sobre deposiciones de esqueletos humanos en zanjas y pozos en la prehistoria reciente de Andalucía", Mainake XXVI: $115-138$.

MÁRQUEZ, J. E. (2006a): "Sobre los depósitos estructurados de animales en yacimientos de fosos del sur de la Península Ibérica", en Weiss-Krejci, E. (coord.), Animais na Pré-História e Arqueologia da Península Ibérica, actas do IV Congresso de Arqueologia Peninsular. Faro, 14-19 de septiembre 2004: 15-25.

MÁRQUEZ, J.E. (2006b): "Neolithic and Copper Age ditched enclosures and social inequality in the Iberian south (IV-III millennia cal BC)", en Díaz-delRío, P. y García Sanjuán, L. (eds.), Social Inequality in Iberian Late Prehistory, BAR International Series XXX.

MÁRQUEZ, J. E. (2007): "La problemática de los recintos de fosos de la Prehistoria Reciente en el sur de España", en Oliveira Jorge, S.; Bettencourt, A. y Figueiral, I. (eds), A concepção das paisagens e dos espaços na Arqueologia da Península Ibérica. Actas do IV Congresso de Arqueologia Peninsular (Faro, Septiembre 2004): 27-35.

MÁRQUEZ, J. E. y FERNÁNDEZ, J. (2002): "Viejos depósitos, nuevas interpretaciones: La Estructura $\mathrm{n}^{\mathrm{o}} 2$ del yacimiento prehistórico de los Villares de Algane (Coín, Málaga)", Mainake XXI-XXII:. 301-333.

MARTÍN DE LA CRUZ, J.C. (1985): Papa Uvas I. Aljaraque, Huelva Campañas de 1976 a 1979. Excavaciones Arqueológicas en España 136, Madrid.

MARTÍN DE LA CRUZ, J.C. (1986): Papa Uvas II. Aljaraque, Huelva Campañas de 1981 a 1983. EAE 149. Madrid.

MARTÍN DE LA CRUZ, J.C. y LUCENA, A. (2003): "Problemas metodológicos e interpretativos que plantean los depósitos sedimentarios del yacimiento arqueológico de Papa Uvas (Aljaraque, Huelva)", Trabalhos de Antropología e Etnologia 43 (1-2): 151-170.

MCGUIRE, R. H. y SCHIFFER, M. B. (1983): “A Theory of Architectural Design", Journal of Anthropological Archaeology 2: 277-303.

MURILLO, M" ${ }^{\mathrm{a}}$. T. (1991): "Excavaciones de urgencia en el poblado calcolítico de Valencina de la Concepción (Sevilla), 1988-1989”, Anuario Arqueológico de Andalucía 1989, III: 555-560.
NOCETE, F. (2001): Tercer milenio antes de nuestra era. Relaciones y contradicciones centro/periferia en el Valle del Guadalquivir. Bellaterra, Barcelona.

RIVERO, E.; CRUZ-AUÑÓN, R. y FERNÁNDEZ, P. (1989): “Avance de los trabajos realizados en el yacimiento de la Edad del Cobre del Negrón (Gilena, Sevilla)", Actas del XIX Congreso Nacional de Arqueología, Castellón, 1987: 329-339. Zaragoza.

ROMÁN, J. M. y CONLIN, E. (2001): “Excavaciones Arqueológicas en el Solar de la Calle Dolores Quintanilla No 6, Carmona (Sevilla)", Anuario Arqueológico de Andalucía 1997, III: 529-535.

RUIZ FERNÁNDEZ, J. A. (1987): “Informe excavaciones de urgencia. Pago de Cantarranas-La Viña. El puerto de Santa María”, Anuario Arqueológico de Andalucía 1986, III: 95-100.

RUIZ FERNÁNDEZ, J. A. y RUIZ GIL, J. A. (1989): "Calcolítico en el Puerto de Santa María", Revista de Arqueología no 94.

RUIZ GIL, J. A. y LÓPEZ AMADOR, J. J (coords.) (2001): Formaciones sociales agropecuarias en la Bahía de Cádiz. 5000 años de adaptación ecológica en la Laguna del Gallo, El Puerto de Santa María. Memoria Arqueológica de Pocito Chico I (19972001), Cádiz.

RUIZ GIL, J. A. y RUIZ MATA, D. (1999): “Cantarranas (El Puerto de Santa María, Cádiz): Un poblado de transición Neolítico Final/Cobre Inicial", II Congrés del Neolitic a la Península Ibérica, Saguntum, extra-2: 223-228.

RUIZ LARA, D. (1987): “Excavación arqueológica de urgencia en "La Minilla" (La Rambla, Córdoba)", Anuario Arqueológico de Andalucía 1986, III: 124-126.

SANTANA, I. (1993): "Excavación arqueológica de urgencia en El Algarrobillo, Valencina de la Concepción (Sevilla)", Anuario Arqueológico de Andalucía 1991, III: 548-553.

SCHIFFER, M. B. (1987): Formation Processes of the Archaeological Record. Albuquerque.

SEYMOUR D. y SCHIFFER, M. (1987): “A Preliminary Analysis of Pithouse Assemblages from Snaketown Arizona", en Kent, S. (ed.), Method and Theory For Activity Area Research. An Etnoarchaeological Approach. New York.

ZAFRA, N.; HORNOS, F. y CASTRO. M. (1999): "Una macro-aldea en el origen del modo de vida campesino: Marroquíes Bajos (Jaén) c. 25002000 cal. ANE", Trabajos de Prehistoria 56, $\mathrm{n}^{\circ} 1$ : 77-102. 